\title{
ON MANIFOLDS WITH THE HOMOTOPY TYPE OF COMPLEX PROJECTIVE SPACE $\left({ }^{1}\right)$
}

\section{BRUCE CONRAD}

\begin{abstract}
It is known that in every even dimension greater than four there are infinitely many nonhomeomorphic smooth manifolds with the homotopy type of complex projective space. In this paper we provide an explicit construction of homotopy complex projective spaces. Our initial data will be a manifold $X$ with the homotopy type of $\mathrm{CP}^{3}$ and an embedding $\gamma_{3}: S^{5} \rightarrow$ $S^{7}$. A homotopy 7 -sphere $\Sigma^{7}$ is constructed and an embedding $\gamma_{4}: \Sigma^{7} \rightarrow S^{9}$ may be chosen. The procedure continues inductively until either an obstruction or the desired dimension is reached; in the latter case the final obstruction is the class of $\sum^{2 n-1}$ in $\Theta_{2 n-1}$. Should this obstruction vanish, the final choice is of a diffeomorphism $\gamma_{n}: \Sigma^{2 n-1} \rightarrow S^{2 n-1}$. There results a manifold, denoted $\left(X, \gamma_{3}, \cdots, \gamma_{n-1}, \gamma_{n}\right)$, with the homotopy type of CPn We describe the obstructions encountered, but are able to evaluate only the primary ones. It is shown that every homotopy complex projective space may be so constructed, and in terms of this construction, necessary and sufficient conditions for two homotopy complex projective spaces to be diffeomorphic are stated.
\end{abstract}

1. Introduction. The study of smooth homotopy complex projective spaces received its first impetus as a means of classifying free circle actions on homotopy spheres [9], [10], [14], [15]. D. Sullivan [17] later classified PL homotopy complex spaces as an application of his characteristic variety theorem. In this paper we examine the geometric structure of smooth homotopy complex projective spaces. Although our methods are specific to this problem, they clarify the relationship between surgery and smoothing obstructions which is central to the general problem of classifying smooth manifolds within a homotopy type.

$\mathbf{C P}$ denotes complex projective space. A point of $\mathbf{C P}^{n}$ is described by homogeneous coordinates $\left[z_{0}, \cdots, z_{n}\right]$, where each $z_{i} \in \mathbf{C}$, and $\left|z_{0}\right|^{2}+\left|z_{1}\right|^{2}+\cdots+$ $\left|z_{n}\right|^{2}=1$. If $\lambda$ is a complex number with $|\lambda|=1$, the points $\left[\lambda z_{0}, \cdots, \lambda z_{n}\right]$ and

Received by the editors June 7, 1971 and, in revised form, January 4, 1972. $57 \mathrm{D} 40$.

AMS (MOS) subject classifications (1970). Primary 57D55; Secondary 57E15, 57C45,

Key words and phrases. Homotopy sphere, transverse regularity, embedding, concordance, framed submanifold, inertial group, surgery, smoothing.

( $\left.{ }^{1}\right)$ The results appearing here are from the author's Ph.D. thesis, written at the University of California, Berkeley, under the supervision of Professor G. E. Bredon, and with the support of the National Science Foundation. 
$\left[z_{0}, \cdots, z_{n}\right]$ are identified. If $i<n, \mathbf{C P} \mathbf{P}^{i}$ is the submanifold of $\mathbf{C P} \mathbf{P}^{n}$ defined by the equation $\left|z_{i+1}\right|^{2}+\cdots+\left|z_{n}\right|^{2}=0$.

Let $\mathbf{P}^{n}$ be the submanifold of $\mathbf{C P}^{n}$ whose points satisfy $\left|z_{0}\right| \leq 1 / 2$. Notice that $\mathbf{C P}^{n}-\mathbf{P}^{n}$ is an open ball of dimension $2 n$, and that $\mathbf{P}^{n}$ has the homotopy type of $\mathbf{C P}^{n-1}$. Consider a homotopy equivalence $f: M \rightarrow \mathbf{P}^{n}$, where $M$ is a smooth $2 n$-dimensional manifold whose boundary is a homotopy sphere (a manifold with such a boundary is called almost closed). In $\$ 3$ we show how to do surgery on $f$ to make bomotopy regular on $\mathbf{P}^{i} \subset \mathbf{P}^{n}, 3 \leq i<n$; that is, $f$ is transverse regular on $\mathbf{P}^{i}$ and, if $M_{i}=f^{-1}\left(\mathbf{P}^{i}\right), f \mid M_{i}$ is a homotopy equivalence onto $\mathbf{P}^{i}$. We call $M_{n-1}$ the desuspension of $M$.

The boundary of each submanifold $M_{i}$ is a homotopy sphere; if we arrange the $M_{i}$ to be nested we can examine the series of codimension two embeddings $\partial M_{i} C$ $\partial M_{i+1}$. Preparing for this, we define in $\$ 2$ certain invariants for such embeddings. The first of these invariants, $\tau$, assigns to $\partial M_{i} \subset \partial M_{i+1}$ the index or Arf invariant of a framed submanifold $W$ of $\partial M_{i+1}$, this $W$ is essentially defined by $\partial W=\partial M_{i}$. If $M_{i+1}^{*}$ is the almost smooth manifold formed by attaching a disc along $\partial M_{i+1}$, $-\tau\left(\partial M_{i}\right)$ becomes the Montgomery-Yang desuspension obstruction for $M_{i+1}^{*}$ [15]. The second invariant, $\delta$, is defined only for embeddings $S^{r} \rightarrow S^{r+2}$, and is useful in comparing two homotopy $\mathbf{P}^{n}$ 's $M$ and $M^{\prime}$ if $M_{n-1}$ is diffeomorphic to $M_{n-1}^{\prime}$.

In $\$ 4$, homotopy $\mathbf{P}^{n}$ 's are constructed. Starting with a homotopy $\mathbf{P}^{n-1}, M$, and an embedding $\gamma: \partial M \rightarrow S^{2 n-1}$, our construction leads to a homotopy $\mathbf{P}^{n}$, $(M, \gamma)$, whose desuspension is $M$. As $\gamma$ runs through the set of embeddings of $\partial M$ in $S^{2 n-1},(M, \gamma)$ runs through the set of suspensions of $M$; the latter set is thus empty if $\partial M \notin b P_{2 n-2}$.

Given two suspensions $(M, \gamma)$ and $\left(M, \gamma^{\prime}\right)$ of a homotopy $\mathbf{P}^{n-1}, M$, we have an embedding $\gamma \#\left(-\gamma^{\prime}\right): \partial M \#(-\partial M)=S^{2 n-3} \rightarrow S^{2 n-1}$. In $\$ 5$, we apply the invariant $\delta$ to this embedding and state necessary and sufficient conditions for the two suspensions to be diffeomorphic. The details may be found in Theorem 4. In $\$ 6$ we apply Theorem 4 to the problem of determining which homotopy $\mathbf{P}^{n}$ 's admit a conjugation, i.e. a diffeomorphism not homotopic to the identity. In the final $\$ 7$ we make some observations about an obstruction theory for smoothing PL homotopy $\mathbf{P}^{n}$ 's; a similar obstruction theory arises from a spectral sequence considered by G. Brumfiel [4].

I wish to thank Professors G. E. Bredon and G. Brumfiel for helpful advice and encouragement, and to acknowledge the influence of [17] in the preparation of this paper.

In what follows $B^{n}$ denotes the open ball, and $D^{n}$ the closed disc in $\mathbf{R}^{n}$. An almost closed manifold with the homotopy type of $\mathrm{P}^{n}$ is an $b P^{n}$. A c-orientation for an $b P^{n}, M$, is a distinguished generator of $H^{2}(M ; Z)$. All $b P^{n}$ 's are c-oriented,

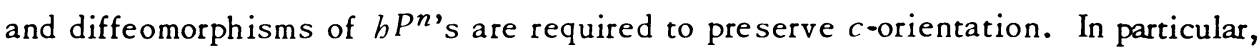


fix as a $c$-orientation for $\mathbf{P}^{n}$ the cohomology class dual to $\left[\mathbf{P}^{n-1}\right] \epsilon$ $H_{2 n-2}\left(\mathbf{P}^{n}, \partial \mathbf{P}^{n} ; \mathbf{Z}\right)$.

2. Invariants for codimension two knots. We present two lemmas which will be useful later. Both proofs center on the evaluation of a certain obstruction which was considered by Kervaire in his proof that every codimension two sphere in $S^{n+2}$ bounds a framed submanifold of $S^{n+2}$; see Theorem I of [11].

We are interested in the following abelian groups:

$G_{n}$, Group of concordance (or $b$-cobordism) classes of embedded homotopy $n$ spheres in $S^{n+2} ;\left({ }^{2}\right)$

$C_{n}$, Group of concordance classes of embeddings of $S^{n}$ in $S^{n+2} ;\left({ }^{2}\right)$

$P{ }_{n}$, Group of framed cobordism classes of framed, almost closed $n$-submanifolds of $D^{n+s}$, s large;

$A_{n}$, Group of almost framed (i.e. framed on the complement of an $n \cdot d i s c$ ) cobordism classes of $n$-submanifolds of $S^{n+s}$.

In each case the group structure is induced by a connected sum operation, e.g. for $P_{n}$ one takes connected sums along the boundary, as in [11]. We will define homomorphisms $\tau: G_{n} \rightarrow P_{n+1}$ and $\delta: C_{n} \rightarrow A_{n+1}$.

If $\Sigma^{n} C S^{n+2}$ is an embedded homotopy sphere whose concordance class is $k \in G_{n}$, find a submanifold $W$ of $S^{n+2}$ with $\partial W=\Sigma^{n}$. Let $v_{1}$ be a normal vector field of $W$ in $S^{n+2}$. By pushing int $(W)$ into int $\left(D^{n+3}\right)$, and letting $F=\left(v_{1}, v_{2}\right)$ where $v_{2}$ is the outward normal vector field of $S^{n+2}$ in $\mathbf{R}^{n+3},(W, F)$ becomes a framed, almost closed submanifold of $D^{n+3}$. Let $\tau(k)$ be the stable equivalence class of $(W, F)$ in $P_{n+1}$.

Now suppose $\sum^{n}$ as above is the image of an embedding $\epsilon: S^{n} \rightarrow S^{n+2}$ and denote by $e$ the concordance class of $\epsilon$ in $C_{n}$. Use $\epsilon$ to attach $D^{n+1}$ to $W$ along $\partial W$ to form an almost framed manifold $(V, F)$ which may be assumed embedded in $S^{n+s}$ for $s$ large. Let $\delta(e)$ be the stable equivalence class of $(V, F)$ in $A_{n+1}$.

Lemma 1. $\tau: G_{n} \rightarrow P_{n+1}$ and $\delta: C_{n} \rightarrow A_{n+1}$ are well-defined epimorphisms if $n \geq 4$.

Proof. If $\Sigma_{0}^{n}, \Sigma_{1}^{n} \subset S^{n+2}$ are concordant there is an $b$-cobordism $C \subset S^{n+2} \times$ $[0,1]$ with $\partial C \cap S^{n+2} \times\{i\}=(-1)^{i} \Sigma_{i}, i=0,1$. Choose $W_{i} \subset D^{n+3}$ with $\partial W_{i}=$ $\Sigma_{i}$, and let $F_{i}$ be the framing of $W_{i}$ constructed as above. $C$ has an essentially unique framing $F_{2}$ in $S^{n+2} \times[0,1]$ extending $(-1)^{i} F_{i} \mid \Sigma_{i}$ on each end. Let $(Y, F)$ be the framed submanifold $\left(W_{0}, F_{0}\right) \cup\left(C, F_{2}\right) \cup-\left(W_{1}, F_{1}\right)$ of $S^{n+3}=$ $D^{n+3} \times\{0\} \cup S^{n+2} \times[0,1] \cup D^{n+3} \times\{1\}$. Choose an open tubular neighborhood $U$ of $Y$; the framing $F$ provides an embedding $j: Y \rightarrow \partial \bar{U}$. Denote by $i$ the inclusion of $\partial \bar{U}$ in $S^{n+3}-U$ and by $k$ the composition $i j: Y \rightarrow S^{n+3}-U$. An argument similar to the proof of $[11$, Theorem I] shows that $(Y, F)$ bounds a framed

$\left(^{2}\right)$ The notations $\theta_{n}^{2}$ for $G_{n}$, and $C_{n}^{2}$ for $C_{n}$, have been used by others. 
submanifold $Q$ of $S^{n+3}$ if $k_{*}[Y]=0$, where $[Y] \in H_{n+1}(Y ; Z)$ is the orientation class: this $Q$ is a framed cobordism between $\left(W_{0}, F_{0}\right)$ and $\left(W_{1}, F_{1}\right)$. Now, $H_{n+1}(\partial \bar{U})=H_{n+1}(Y) \otimes H_{0}\left(S^{1}\right) \oplus H_{n}(Y) \otimes H_{1}\left(S^{1}\right)$. Thus write $j_{*}[Y]=([Y] \otimes 1$, $\left.\alpha \otimes\left[S^{1}\right]\right)$, with $a \in H_{n}(Y)$, and observe that our choice of framing implies $\alpha=0$. Therefore $k_{*}[Y]=i_{*}([Y] \otimes 1,0)$ which is zero by the homology sequence of the pair $\left(S^{n+3}-U, \partial \bar{U}\right)$.

To see that $\delta$ is well defined, let $\epsilon_{0}, \epsilon_{1}$ be concordant embeddings of $S^{n}$ in $S^{n+2}$. Then there is an embedding $E: S^{n} \times[0,1] \rightarrow S^{n+2} \times[0,1]$ such that $E \mid S^{n} \times\{i\}=\epsilon_{i}$. Putting $\Sigma_{i}=\epsilon_{i}\left(S^{n}\right)$, and $C=E\left(S^{n} \times[0,1]\right)$, we construct $\left(W_{i}, F_{i}\right)$, $Y$, and $Q$ as above. By attaching $D^{n+1} \times[0,1]$ to $Q$ along $C$ with $E$, we can construct an almost framed cobordism between $\left(V_{0}, F_{0}\right)$ and $\left(V_{1}, F_{1}\right)$, where $V_{i}=$ $D^{n+1} \cup_{\epsilon_{i}} W_{i}$

A manifold representing a generator of $P_{n+1}$ can be embedded in $S^{n+2}$ as in [6]: thus $\tau$ is onto. By chasing the diagram (exact rows):

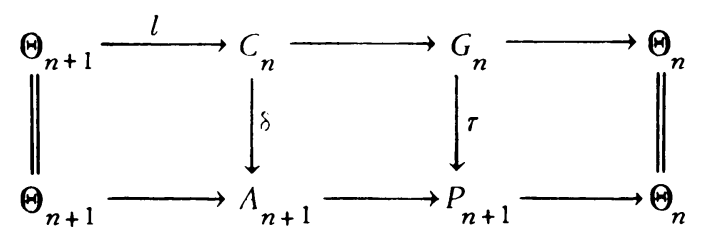

we see that $\delta$ is onto. $l: \Theta_{n+1} \rightarrow C_{n}$ is defined as follows: Let $j: S^{n} \rightarrow S^{n+2}$ be the standard embedding, and $\omega$ be a diffeomorphism of $S^{n}$. If $[\omega]$ is the isotopy class of $\omega$, viewed as an element of $\Theta_{n+1}$, let $l([\omega])$ be the concordance class of $j \omega$. The top exact sequence originated in [7].

Let $S^{n+2}=H_{+} \cup H_{-}$be the union of two hemispheres, with $H_{+} \cap H_{-}=$ $S^{n+1}$. Suppose $\Sigma_{1}^{n}, \Sigma_{2}^{n}$ are embedded homotopy spheres, with $H_{+} \cap \Sigma_{1}, H_{-} \cap \Sigma_{2}$ unknotted and $\Sigma_{1} \cap S^{n+1}=\Sigma_{2} \cap S^{n+1}$, with both intersections transverse; then $\Sigma_{1} \# \Sigma_{2}=\left(H_{-} \cap \Sigma_{1}\right) \cup\left(H_{+} \cap \Sigma_{2}\right)$. Let $g: \Sigma_{2} \rightarrow S^{n}$ be a degree one map, with $g \mid\left(H_{-} \cap \Sigma_{2}\right)=$ (identity), so that $g: \Sigma_{1} \# \Sigma_{2} \rightarrow \Sigma_{1}$ is defined.

Lemma 2. $g: \Sigma_{1} \# \Sigma_{2} \rightarrow \Sigma_{1}$ extends to a degree one map $f: S^{n+2} \rightarrow s^{n+2}$, transverse regular on $\Sigma_{1}$, with $f^{-1}\left(\Sigma_{1}\right)=\Sigma_{1} \# \Sigma_{2}$.

Proof. First suppose $\Sigma_{1}$ is the standard $S^{n} C S^{n+2}$. Let $U$ be a closed tubular neighborhood of $\Sigma_{2}$, and $T$ be a closed tubular neighborhood of $\Sigma_{1}$; then $g$ extends to a fibre-preserving map $G: U, \partial U \rightarrow T, \partial T$. Put $C=\mathrm{Cl}\left(S^{n+2}-T\right)$; then $C \simeq S^{1}$ and we must extend $G \mid \partial U: \partial U \rightarrow C$ over $\mathrm{Cl}\left(S^{n+2}-U\right)$. The obstruction to such an extension vanishes as in $[11$, loc. cit.].

The general case now follows easily. If $f$ is constructed as above, notice that since $\Sigma_{2} \cap H_{-}$is unknotted we may require $/ H_{-}$to be the identity; then $f^{-1}\left(\Sigma_{1}\right)=\Sigma_{1} \# \Sigma_{2}$. 
3. Desuspensions. If $f: M, \partial M \rightarrow \mathbf{P}^{n}, \partial \mathbf{P}^{n}$ is a homotopy equivalence, we say that $f$ is admissible if $f \mid \partial M$ is homotopy regular on $\partial \mathbf{P}^{n-1}$ and if $f$ is admis. sible we say $f$ desuspends if $f \simeq g$ rel $\partial M$ where $g$ is homotopy regular on $\mathbf{P}^{n-1}$.

Montgomery and Yang have dealt in [15] with the problem of desuspending homotopy equivalences $f: N \rightarrow \mathbf{C} \mathbf{P}^{n}$, where $N$ is a closed manifold (such a map desuspends if it is homotopic to $g$ with $g$ homotopy regular on $\mathbf{C P}^{n-1}$ ). They found that surgery could be applied to $f$, with an obstruction in the "middle dimension." Thus $f \simeq b$ such that $b$ is transverse regular on $\mathbf{C P}^{n-1}$ and $b_{*}: \pi_{i}\left(b^{-1} \mathbf{C P}^{n-1}\right) \rightarrow$ $\pi_{i}\left(\mathbf{C} \mathbf{P}^{n-1}\right)$ is an isomorphism if $i<n-1$. Put $L=b^{-1}\left(\mathbf{C} \mathbf{P}^{n-1}\right)$, and $K_{n-1}(b)=$ $\operatorname{Ker} b_{*}: H_{n-1}(L) \rightarrow H_{n-1}\left(C^{n-1}\right)$. The obstruction to desuspending $f$ is then the index of the (symmetric) intersection form on $K_{n-1}(b)$ when $n$ is odd. In case $n$ is even the intersection form is antisymmetric and so admits a symplectic basis. Associated to this basis is an Arf invariant which is the desuspension obstruc. tion. In either case, we denote the desuspension obstruction by $\mathcal{S}(N, f) ; \mathcal{S}(N, f) \in$ $\mathbf{Z}$ if $n$ is odd and $\mathbf{Z}_{2}$ if $n$ is even.

The obstruction to desuspending an admissible homotopy equivalence $f: M$, $\partial M \rightarrow \mathbf{P}^{n}, \partial \mathbf{P}^{n}$ arises from the same argument and has values in the same groups. Of course it depends on $f \mid \partial M$; we emphasize this by denoting it $\mathcal{S}(M, \partial M, f)$.

If $M$ is an almost closed manifold, let $M^{*}$ be the PL manifold constructed by attaching a cone to the boundary of a $C^{\infty}$ triangulation of $M$. The following theorem relates the two obstructions $\delta\left(M^{*}, F\right)$ and $\delta(M, \partial M, f)$, when $F$ is the extension to $M^{*}$ of $f: M \rightarrow \mathrm{P}^{n} \subset \mathbf{C P}$.

Theorem 1. Let $f: M, \partial M \rightarrow \mathbf{P}^{n}, \partial \mathbf{P}^{n}$ be an admissible bomotopy equivalence, $n>3$. fdesuspends iff $\delta(M, \partial M, f)=0$. Furthermore,

$$
\mathcal{S}(M, \partial M, f)=\mathcal{S}\left(M^{*}, F\right)+\tau\left(f^{-1}\left(\partial \mathbf{P}^{n-1}\right) \subset \partial M\right),
$$

Notice that $\tau\left(f^{-1}\left(\partial \mathbf{P}^{n-1}\right) \subset \partial M\right) \in P_{2 n-2}$ which we may identify with $\mathrm{Z}_{2}$ if $n$ is even, and $8 Z$ if $n$ is odd using the isomorphisms described in [12]. These identifications are implicit in (1).

Proof of Theorem 1. $\delta(M, \partial M, f)=0$ iff $f$ desuspends is proved by straightforward modification of [15].

To prove (1), triangulate $M$ and $\mathbf{P}^{n}$ and suppose $f$ is an admissible PL homotopy equivalence which is transverse regular on $\mathrm{P}^{n-1}$. Putting $L=f^{-1}\left(P^{n-1}\right)$ we may suppose $f \mid L$ is $(n-1)$-connected. Put $K_{n-1}(f)=\operatorname{Ker} f_{*}: H_{n-1}(L) \rightarrow$ $H_{n-1}\left(\mathbf{P}^{n-1}\right)$. Again, by performing a homotopy rel $M$ if necessary, we may assume $F$ is transverse regular on $\mathbf{C P}^{n=1}$, with $F^{-1}\left(\mathbf{C P}^{n-1}\right)=L \cup Q ; Q \subset \mathrm{Cl}\left(M^{*}-M\right)$ transverse to $\partial M$. Additionally, we suppose $Q$ is $(n-2)$-connected. Then $K_{n-1}(F) \cong H_{n-1}(Q) \oplus K_{n=1}(f)$, so $\delta\left(M^{*}, F\right)$ is the sum of the index or Arf invariants of $H_{n-1}(Q)$ and $K_{n-1}(f)$. The latter quantity is $\delta(M, \partial M, f)$. Since there is the relation of knots $(\partial L \subset \partial M)=-(\partial Q \subset \partial M)$ the index or Arf invariant of $H_{n-1}(Q)$ is $-\tau(\partial L \subset \partial M)=-\tau\left(f^{-1}\left(\partial \mathrm{P}^{n-1}\right) \subset \partial M\right)$. 
Corollary 1 . If $M$ is an $b P^{n}, n>3$, there is an admissible bomotopy equivalence $f: M, \partial M \rightarrow \mathbf{P}^{n}, \partial \mathbf{P}^{n}$ which desuspends.

Proof. Choose a c-orientation-preserving homotopy equivalence $g: M^{*} \rightarrow$ $\mathrm{CP}^{n}$, and let $\Sigma \subset \partial M$ be a knot such that $\tau(\Sigma)=-\delta\left(M^{*}, g\right)$. Lemma 2 provides a degree one map $p: \partial M \rightarrow S^{2 n-1}$, transverse regular on $S^{2 n-3}$, such that $p^{-1}\left(S^{2 n-3}\right)=\Sigma$, and we can extend $p$ to an admissible $c$-orientation-preserving homotopy equivalence $f: M, \partial M \rightarrow \mathbf{P}^{n}, \partial \mathbf{P}^{n}$. Applying $(1), \mathcal{S}(M, \partial M, f)=0$ so $f$ desuspends.

If $f: M, \partial M \rightarrow \mathbf{P}^{n}, \partial \mathbf{P}^{n}$ is a c-orientation-preserving homotopy equivalence which is homotopy regular on $\mathbf{P}^{n-1}, f^{-1}\left(\mathbf{P}^{n-1}\right)$ with the induced $c$-orientation is said to be a desuspension of $M$. Standard relative surgery arguments show that desuspensions are unique in the following sense:

Theorem 2. Let $P$ and $Q$ be desuspensions of the $b P^{n}, M$. Then there is a diffeomorphism $f$ of $M$ such that $f$ is pseudo-isotopic to the identity and $f(P)=Q$.

4. Suspensions. If $M$ is an $b P^{n}, n>3$, with $\partial M \in b P_{2 n}$, we are going to show how to construct suspensions of $M$ : that is, $b P^{n+1} s$ whose desuspensions will be $M$.

Let $\xi_{M}$ be the 2-plane bundle over $M$ whose Euler class is the $c$-orientation of $M . E_{M}$ and $S_{M}$ are to denote the total spaces of the associated $D^{2}$ and $S^{1}$. bundles, and $E_{\partial M}, S_{\partial M}$ their restrictions to $\partial M$.

Lemma 3. $S_{M}$ is diffeomorphic to $\partial M \times D^{2}$.

Proof. Let $S_{M^{*}}$ be the extension of $S_{M}$ over the PL homotopy complex projective space $M^{*} \cdot S_{M^{*}}$ is homeomorphic to $S^{2 n+1}$, and $S_{M^{*}}-S_{M}$ is homeomorphic to $S^{1} \times B^{2 n}$; since $S^{1}$ must be unknotted in $S^{2 n+1}$, we see that $S_{M}$ is a smooth manifold which is homeomorphic to $S^{2 n-1} \times D^{2}$, and whose boundary $S_{\partial M}$ is diffeomorphic to $\partial M \times S^{1}$. $\operatorname{Int}\left(S_{M}\right)$ is a smoothing of $S^{2 n-1} \times B^{2}$; by the product theorem of Hirsch [8] there is a unique (up to diffeomorphism) homotopy sphere $\Sigma$ such that $\Sigma \times B^{2}$ is concordant to $\operatorname{int}\left(S_{M}\right)$. It will be sufficient to show that $\Sigma \approx \partial M$. The complement of a small open tubular neighborhood of $\Sigma \times\{0\}$ in $S_{M}$ is easily seen to be an $b$-cobordism between $\Sigma \times S^{1}$ and $\partial M \times S^{1}$; since $W b(Z)=$ 0 th is $b$-cobordism is a product. According to [2], two homotopy spheres are diffeomorphic if their products with $S^{1}$ are diffeomorphic: therefore $\Sigma \approx \partial M$.

Now choose an embedding $\gamma: \partial M \rightarrow S^{2 n+1}$ (recall that such embeddings exist iff $\partial M \in b P_{2 n}$ ). Let $\Gamma: \partial M \times D^{2} \rightarrow S^{2 n+1}$ be a collar for $\gamma, \alpha: E_{\partial M} \rightarrow \partial M \times D^{2}$ be a trivialisation, and $\sigma: S_{\partial M} \rightarrow \partial M \times S^{1}$ be the restriction of $\alpha$. From the disjoint union $E_{M}\left(S^{2 n+1} \times[0,1]\right.$ form a manifold $X$ by identifying $x \in E_{\partial M}$ with $(y \alpha(x), 0) \in S^{2 n+1} \times\{0\}$ and straightening the angle. The two components, $\partial_{0} X=$ $S_{M} \cup_{\Gamma \sigma}\left(S^{2 n+1} \times\{0\}-\Gamma\left(\partial M \times B^{2}\right)\right)$ and $\partial_{1} X=S^{2 n+1} \times\{1\}$, of $\partial X$ are homotopy spheres; compare Lemma 3. 
Lemma 4. The diffeomorphism class of $X$ is independent of the choice of $\gamma$ within its concordance class.

Proof. Let $\gamma_{0}, \gamma_{1}$ be concordant embeddings of $\partial M$ in $S^{2 n+1}$, and choose collars $\Gamma_{0}, \Gamma_{1}: \partial M \times D^{2} \rightarrow S^{2 n+1}$. Let $v$ be a concordance between $\gamma_{0}$ and $\gamma_{1}$, parameterized so that $v: \partial M \times[0,1] \rightarrow S^{2 n+1} \times[-1,0]$ with $v \mid \partial M \times\{i\}=\left(\gamma_{i}\right.$, $i-1)$, for $i=0,1$. There is no obstruction to extending $v$ to a concordance $\Upsilon$ between $\Gamma_{0}$ and $\Gamma_{1}$, so we assume this is done.

Let $2 D^{2} \subset \mathbf{R}^{2}$ be the disc of radius 2 , and $2 E_{M}$ be the $2 D^{2}$-bundle associated with $\xi_{M}$. Construct the manifold $2 X_{0}$ from $2 E_{M} \cup S^{2 n+1} \times[-1,1]$ by identifying $x \in 2 E_{\partial M}$ with $(\Gamma \alpha(1 / 2 x),-1) .2 X_{0}$ is obviously diffeomorphic to $X_{0}$, and embedded in $2 X_{0}$ we will discover a copy of $X_{1}$ as follows.

Choose a collar $K$ of $\partial M$ in $M$, and a diffeomorphism $\beta: E_{K} \rightarrow \partial M \times D^{2} \times$ $[0,1]$ such that $\beta \mid E_{\partial M}=(\alpha, 1)$. Also choose a diffeomorphism $j: \mathrm{Cl}\left(E_{M}-E_{K}\right) \rightarrow$ $E_{M}$ such that where both are defined $\beta=(\alpha j, 0)$, and define $k: E_{M} \rightarrow 2 X_{0}$ by $k(x)=j(x)$ if $x \in \mathrm{Cl}\left(E_{M}-E_{K}\right)$ and $k(x)=(\beta(1 / 2 x))$ if $x \in E_{K}$. Then $X_{1} \approx k\left(E_{M}\right) \cup$ $S^{2 n+1} \times[0,1]$. Moreover, upon straightening the angles

$$
\mathrm{Cl}\left(2 X_{0}-X_{1}\right)=S_{M} \times[1,2] \cup\left(S^{2 n+1} \times[-1,0]-\Upsilon\left(\partial M \times 1 / 2 B^{2} \times[0,1]\right)\right)
$$

becomes an $b$-cobordism between $\partial_{0} X_{0}$ and $\partial_{0} X_{1}$; thus $X_{0} \approx X_{1}$.

Let $i: M, \partial M \rightarrow X, \partial_{1} X$ be defined as the composition $M \stackrel{d}{\rightarrow} M \cup \partial M \times[0,1] \stackrel{e}{\rightarrow}$ $X$ where $d$ is a diffeomorphism that is the identity outside a collar of $\partial M$, and

$$
e=(0 \text {-section }) \cup \gamma \times \text { id }: M \cup \partial M \times[n, 1] \rightarrow E_{M} \cup S^{2 n+1} \times[0,1] .
$$

We could attach $D^{2 n+2}$ to $X$ along $\partial_{1} X$; while the resulting almost closed manifold would be an $h P^{n+1}$, its desuspension would not be $i(M)$. We therefore prefer to make a slight alteration in the differential structure of $M$. Specifically, let $B \subset \partial_{0} X$ be an open ball, and let $\iota: \bar{B} \rightarrow S^{2 n+1}-\mathrm{im}(\Gamma)$ be an orientation. preserving embedding. Putting

$$
X^{\prime \prime}=(X-(\iota(B) \times[0,1])) \cup_{\iota}\left(\partial_{0} X-B\right) \times[0,1],
$$

$\partial_{0} X^{\prime}=\left(\partial_{0} X\right) \#\left(-\partial_{0} X\right) \approx s^{2 n+1}$ and $\partial_{1} X^{\prime} \approx-\partial_{0} X$.

Let $(M, \gamma)$ be the almost closed manifold obtained by attaching $D^{2 n+2}$ to $X^{\prime}$ a long $\partial_{0} X^{0}$. It is clear that the diffeomorphism class of $(M, \gamma)$ is dependent only on the diffeomorphism class of $M$ and the concordance class of $\gamma$.

Since $(M, \gamma)$ is obtained from $E_{M}$ by attaching a cell along $S_{M}$ it is obvious that $(M, \gamma)$ is an $b P^{n+1}$. This fact, together with Corollary 1 and Theorem 2, gives us the following theorem:

Theorem 3. $(M, \gamma)$ is an $b P^{n+1}$. Moreover, if $N$ is any $b P^{n+1}$ there exists a unique $b P^{n}, M$, and an embedding $\gamma: \partial M \rightarrow S^{2 n+1} \operatorname{sucb}$ that $(M, \gamma) \approx N$. 
Let $\Xi_{n}$ be the set of $c$-oriented diffeomorphism classes of $b P^{n}$,s and $\Xi_{n}(\partial \pi)$ be the subset consisting of elements whose boundaries belong to $b P_{2 n}$. There is a desuspension function $d: \Xi_{n+1} \rightarrow \Xi_{n}(\partial \pi)$ defined by $d(M, \gamma)=M$.

Corollary 2. There is an action of $C_{2 n-1}$ on $\Xi_{n+1}$. The orbit set of this action is placed in 1-1 correspondence with $\Xi_{n}(\partial \pi)$ by the desuspension function.

Proof. $C_{2 n-1}$ acts transitively on the set of concordance classes of embeddings of $\partial M$ in $S^{2 n+1}$ by a connected sum operation \#. Thus, if $N=(M, \gamma) \epsilon$ $\Xi_{n+1}$ and $e \in C_{2 n-1}$, let $e \cdot N=(M, e \# \gamma)$.

Remark. We can continue to decompose an $b P^{n+1}, N$ until we arrive at a sequence $\left(W, \gamma_{3}, \cdots, \gamma_{n}\right)$, where $W$ is an $b P^{3}$ and $\gamma_{i}: \Sigma^{2 i-1} \rightarrow S^{2 i+1}$ is an embedding of the homotopy sphere $\partial\left(\left(\left(\cdots\left(W, \gamma_{3}\right), \gamma_{4}\right) \cdots\right), \gamma_{i-1}\right)=\Sigma$; this sequence determines the diffeomorphism class of $N$. Omitting certain parentheses from our notation, we shall write $N \approx\left(W, \gamma_{3}, \cdots, \gamma_{n}\right)$.

5. Determination of the action of $C_{2 n-1}$ on $\Xi_{n+1}$. It is a consequence of [17], [18] that $\Xi_{n+1}$ is in $1-1$ correspondence with $\left[\mathbf{P}^{n+1} ; F / 0\right] \simeq[\mathbf{C P}, F / 0]$. Moreover, the desuspension $d: \Xi_{n+1} \rightarrow \Xi_{n}$ corresponds to $i^{\#}$ in the Puppe sequence

(2) $\left[S \mathrm{CP}^{n-1} ; F / 0\right] \stackrel{k^{\#}}{\longrightarrow}\left[\mathbf{C P} \mathbf{P}^{n} / \mathbf{C P}^{n-1} ; F / 0\right] \stackrel{j^{\#}}{\longrightarrow}\left[\mathbf{C P}^{n} ; F / 0\right] \stackrel{i^{\#}}{\longrightarrow}\left[\mathbf{C P}^{n-1} ; F / 0\right]$.

Now, $\left[\mathrm{CP}^{n} / \mathrm{CP}^{n-1} ; F / 0\right] \cong \pi_{2 n}(F / 0) \cong A_{2 n}$ so $j^{\#}$ induces an action of $A_{2 n}$ on $\left[\mathrm{CP}^{n} ; F / 0\right]$. We will show that our action of $C_{2 n-1}$ on $\Xi_{n+1}$ factors through this action via the homomorphism $\delta$ of $\$ 2$. Upon identifying $\operatorname{Im} k^{\#}$ with the inertial group $I\left(\mathbf{C P}^{n}\right)$ we will then have a complete description of the action of $C_{2 n-1}$ on $\Xi_{n+1}$; or equivalently a necessary and sufficient condition for two suspensions of the same $b P^{n}$ to the diffeomorphic, as follows. Let $I_{n} \subset \Theta_{2 n}$ be the inertial group of $\mathbf{C P}$.

Theorem 4. If $e \in C_{2 n-1}$ and $M \in \Xi_{n+1}, e \cdot M \approx M$ iff $\delta(e) \in I_{n}$.

Theorem $4^{\prime}$. If $M$ is an $b P^{n}$ and $\gamma, \gamma^{\prime}: \partial M \rightarrow S^{2 n+1}$ are embeddings, $(M, \gamma) \approx$ $\left(M, \gamma^{\prime}\right)$ iff $\gamma \#\left(-\gamma^{\prime}\right):(\partial M) \#(-\partial M) \approx s^{2 n-1} \rightarrow s^{2 n+1}$ satisfies $\delta\left(\gamma \#\left(-\gamma^{\prime}\right)\right) \in I_{n^{\circ}}$ $A_{2 n}$.

Remark. Note that $\Theta_{2 n} \rightarrow A_{2 n}$ is injective since $b P_{2 n+1}=0$. Thus $I_{n} C$

Let $\alpha: \pi_{2 n}(F / 0) \rightarrow A_{2 n}$ be the isomorphism defined by Sullivan in [17]. We define $\delta^{\prime}: C_{2 n-1} \rightarrow \pi_{2 n}(F / 0)$ so that $\delta=a \delta^{\prime}$. Using the definition of $\delta^{\prime}$, it is a simple matter to factor the action of $C_{2 n-1}$ on $\Xi_{n+1}$ through $A_{2 n}$ as promised above.

If $\epsilon: S^{m-1} \rightarrow S^{m+1}$ is an embedding, let $\epsilon^{\prime}: S^{m-1} \times D^{2} \rightarrow S^{m+1}$ be a collar for $\epsilon$. Choose a smooth degree 1 map $f: S^{m+1} \rightarrow S^{m+1}$ with $f^{-1}\left(S^{m-1}\right)=\epsilon\left(S^{m-1}\right)$ and such that $f \epsilon^{\prime}: S^{m-1} \times D^{2} \rightarrow S^{m+1}$ is the standard embedding of $S^{m-1} \times D^{2}$ 
in $S^{m+1}$; such a map exists by Lemma 2. Extend $f$ to $F: D^{m+2} \rightarrow D^{m+2}$ and let $\pi:\left(D^{m+2}, S^{m-1}\right) \rightarrow\left(D^{m}, S^{m-1}\right)$ be the composition

$$
\left(D^{m+2}, S^{m-1}\right) \rightarrow\left(D^{m} \times D^{2}, S^{m-1} \times\{0\}\right) \rightarrow\left(D^{m}, S^{m-1}\right) .
$$

By construction, $\pi f \epsilon^{\prime}: S^{m-1} \times D^{2} \rightarrow S^{m-1}$ is the projection. Let $E=\left(B^{m+2} \cup\right.$ $\left.\epsilon^{\prime}\left(S^{m-1} \times B^{2}\right)\right) \times R^{s-2}, s$ large, and define $t: E \rightarrow D^{m} \times R^{s}$ by $t(x, y)=(F(x), y)$, where we have identified $\left(B^{m+2} \cup S^{m-1} \times B^{2}\right) \times R^{s-2}$ with $D^{m} \times R^{s}$. Now let $i: D^{m} \rightarrow E$ be an embedding extending $(\epsilon, 0): S^{m-1} \rightarrow E$. E fibres over $i\left(D^{m}\right)$ as an extension of the trivial fibration $\pi f: \epsilon^{\prime}\left(S^{m-1} \times B^{2}\right) \rightarrow S^{m-1}$, and $t$ is a fibre homotopy trivialisation of this fibration. The element of $\left[\left(D^{m}, S^{m-1}\right) ; F / 0, *\right]$ $=\pi_{m}(F / 0)$ defined by $(E, t, \pi f)$ is $\delta^{\prime}(\epsilon)$. It is clear from the definition of $\alpha$ that $\delta=\alpha \delta^{\prime}$.

If $(M, \gamma)=N$ is an $b P^{n+1}$, and $e \in C_{2 n-1}$, there is a homotopy equivalence $b: e \cdot N \rightarrow N$, defined as follows. Let $\epsilon: S^{2 n-1} \rightarrow S^{2 n+1}$ be an embedding representing $e$. Then $e \cdot N=(M, \gamma \# \epsilon)$. By Lemma 2 we can find a degree one map $f: \partial(e \cdot N) \rightarrow \mathrm{N}$ such that $f^{-1}(\gamma(\partial M))=\gamma \# \epsilon(\partial M)$. Mapping $E_{M}$ by the identity and $\partial(e \cdot N) \times I$ by $f$, we may extend without obstruction to the homotopy equivalence $b$.

The following lemma establishes the factorization of the action of $C_{2 n-1}$ on $\Xi_{n+1}$ through $A_{2 n}$.

Lemma 5. With the above notation, the characteristic $F / 0$ bundle [17] for $h$, $\theta_{b}=-j^{\#} \delta^{\prime}(e) \in[N ; F / 0]$.

Proof. Since $h \mid M$ is the identity, $\theta_{b} \in \operatorname{Im} j^{\#}:[(N, M) ; F / 0, *] \rightarrow[N ; F / 0]$. The proof that $\theta_{b}=j^{\sharp}\left(-\delta^{0}(e)\right)$ is straightforward and omitted.

If $V^{m}$ is a closed manifold recall that the inertial group $I\left(V^{m}\right)$ is the subgroup of $\Theta_{m}$ consisting of elements $\sigma$ such that $\sigma \# V^{m}$ is diffeomorphic to $V^{m}$ by a diffeomorphism homotopic to the identity. We propose to extend this definition to cover the case of almost closed manifolds.

Diffeomorphisms $f_{0}, f_{1}$ of a closed manifold $W^{m-1}$ are conjugate if there is a diffeomorphism $g$ of $W$ such that $f_{0}$ is pseudo-isotopic to $g f_{1} g^{-1}$ [3]. Let $\mathscr{D}_{0}\left(W^{m-1}\right)$ be the set of conjugacy classes of diffeomorphisms of $W$ which are homotopic to the identity. As in [3] we have a natural map $p_{W}: \Theta_{m} \rightarrow \mathscr{D}_{0}\left(W^{m-1}\right)$.

Now let $V^{m}$ be a manifold with connected boundary $W$. The inertial group $I(V)$ of $V$ is then defined to be the subgroup of $\Theta_{m}$ consisting of elements $\alpha$ such that $p_{W}(\alpha)$ extends to a diffeomorphism of $V$ which is homotopic to the identity. If $V=M-$ (open disc), where $M$ is a closed manifold, then $I(V)=I(M)$, the latter group being defined in the usual way. The following theorem is a first step toward identifying the image of $k^{\#}$ in (2).

Theorem 5. Let $M$ be an $b P^{n}$ with $\partial M \in b P_{2 n^{*}}$ Then $I(M)=I_{n}$.

The proof is contained in the following three lemmas. 
Lemma 6. If $W^{m-1}$ is a bomotopy sphere which bounds a $\pi$-manifold then $p_{W}$ is an isomorphism.

Proof. This lemma is well known; however, a proof is included for completeness. For any homotopy sphere $W, p_{W}$ is onto by an obvious argument. By [3, Lemma 1], $\operatorname{Ker} p_{W}=I\left(W \times S^{1}\right)$. But $I\left(W \times S^{1}\right)=\{0, \eta \cdot\{W\}\}$ where $\eta$ generates the stable stem $\pi_{1},\{W\} \in \Theta_{m-1}$ and the action of $\pi_{*}$ on $\Theta_{*}$ is in [1]; see [5, Theorem 3.1]. But since $W$ is a $\pi$-boundary, $\eta \cdot\{W\}$ is clearly 0 .

Let $G$ denote the circle group of complex numbers with unit modulus. If $\phi$ is a $G$-action on a homotopy sphere $W^{m}$, we can consider the Hopf construction on $\phi: G \times W \rightarrow W$, which yields an element $b_{\phi} \in[G * W ; S W]=\pi_{m+2}\left(S^{m+1}\right)$. We say that the action $\phi$ is essential if $b_{\phi} \neq 0$.

Lemma 7. If $W^{m}$ bounds a $\pi$-manifold, then $W$ admits an essential G-action.

Proof. If $m$ is even, then $W \approx S^{m}=\left\{(z, \mathbf{x}) \in \mathbf{C} \times \mathbf{R}^{m-1}:|z|^{2}+\|x\|^{2}=1\right\}$. Define the $G$-action $\phi$ by $\phi(g,(z, \mathbf{x}))=(g z, \mathbf{x})$. Then $b_{\phi}$ is the $(m-1)$-fold suspension of the Hopf map $b: S^{3} \rightarrow S^{2}$ and is thus essential.

If $m=4 k+1$, then $W$ is diffeomorphic to the Brieskorn variety $V_{d} \subset C^{2 k+2}$ defined by the equations

$$
\left|z_{0}\right|^{2}+\cdots+\left|z_{2 k+1}\right|^{2}=1 ; \text { and } z_{0}^{d}+z_{1}^{2}+\cdots+z_{2 k+1}^{2}=0, \text { for } d \text { odd. }
$$

In this case, define $\phi$ by $\phi(g, z)=\left(g^{2} z_{0}, g^{d} z_{1}, \cdots, g^{d} z_{2 k+1}\right)$. Clearly $\phi$ is essential on $S^{4 k+3}$ and leaves $V_{d}$ invariant. Principal orbits in $S^{4 k+3}-V_{d}$ link $V_{d}(2 d)$ times; therefore $\phi \mid V_{d}$ is essential as well.

If $m=4 k-1$ we can define in a similar fashion essential $G$-actions on the Brieskorn varieties $V_{p, q} \subset C^{2 k+1}$ with equations $\|z\|=1$; and $z_{0}^{p}+z_{1}^{q}+z_{2}^{2}+\cdots+z_{2 k}^{2}=0, \quad$ for $(2, p, q)$ pairwise relatively prime; the details are left to the reader. Since $W$ must be diffeomorphic to some $V_{p, q}$, this completes the proof.

Remark. R. Schultz has shown that if a homotopy sphere $W$ admits an essential circle action then $\eta \cdot\{W\}=0$ [16]. It would be interesting to know if this condition is sufficient.

Now let $W^{2 n-1}$ be a homotopy sphere which bounds a $\pi$-manifold and let $\omega$ be a diffeomorphism of $W$. As in [3], to each homotopy $b$ of $\omega$ with the identity there corresponds an element $\theta_{b, \omega} \in[S W ; F / 0]$. Lemma 7 implies that $\theta_{b, \omega}$ is independent of $b$, as follows:

If $b^{\prime}$ is another homotopy, $\theta_{b, \omega}=\theta_{b^{\prime}, \omega}$ iff there is a diffeomorphism $f$ of $W \times I$ with $f \mid W \times \dot{I}=$ id and $b^{\prime} f \simeq b$ rel $W^{\prime} \times \stackrel{\omega}{\dot{I}}$. But if $\phi$ is an essential $S^{1}$ action on $W$, we could put $f(x, t)=(\phi(t, x), t)$. Then either $b^{\prime} \simeq b \operatorname{rel} W \times i$ or $b^{\prime} f \simeq$ $b$ rel $W \times \dot{i}$.

Thus we simply write $\theta(\omega)$ for the element of $\pi_{2 n}(F / 0)$ corresponding to $\omega$. 
Lemma 8. Let $M$ be an $b P^{n}$ with $\{\partial M\} \in b P_{2 n}$, and let $r: \partial M \rightarrow M$ denote the inclusion. Then $\theta \circ p_{\partial M}$ places $I(M)$ in $1-1$ correspondence with $\operatorname{Im} r^{\#}:[S M ; F / 0] \rightarrow$ $[S \partial M ; F / 0]$.

Proof. Suppose $\omega$ is a diffeomorphism of $\partial M$ and $\theta(\omega)=r^{\sharp} \eta$. The problem of finding a diffeomorphism of $M$ extending $\omega$ is essentially that of finding a homotopy equivalence of manifolds $T, \partial T \rightarrow M \times S^{1}, \partial M \times S^{1}$ such that $\partial T$ is the mapping torus of $\omega$; see [3]. But since $\operatorname{dim} M \times S^{1}$ is odd, there is no obstruction to doing surgery rel $\partial M \times S^{1}$ in the $F / 0$-bundle $c^{*} \eta$, where $c: M \times S^{1} \rightarrow M \wedge S^{1}=S M$ is the collapsing map, to get such a homotopy equivalence.

To complete the proof, we show $\theta p_{\partial M}(I(M)) \subset \operatorname{Im} r^{\#}$. Let $\Omega$ be a diffeomorphism of $M$ and $b_{t}$ be a homotopy of $\Omega$ with the identity. The obstructions to altering $b_{t}: M \times I \rightarrow M$ by a homotopy rel $M \times\{0,1\}$, to obtain a new homotopy $k_{t}$ with $k_{t}(\partial M) \subset \partial M$ lie in $H^{i}\left(\partial M \times I, \partial M \times\{0,1\} ; \pi_{i}(M, \partial M)\right)=H^{i-1}\left(\partial M, \pi_{i}(M, \partial M)\right)=0$ since $\pi_{i}(M, \partial M)=0$ for $i \neq 2$. Thus $r^{\#} \theta\left(\Omega, k_{t}\right)=\theta(\Omega \mid \partial M)$.

We are now ready to prove Theorem 4. Suppose first that $e \in C_{2 n-1}$ with $\delta(e) \in I_{n}$. Then there is a diffeomorphism $\omega$ of $S^{2 n-1}$ such that for an $b P^{n+1} N=$ $(M, \gamma), e \cdot N=(M, \gamma \# j \omega)$ where $j: S^{2 n-1} \rightarrow S^{2 n+1}$ is the standard embedding. If $\tilde{\omega}$ is a diffeomorphism of $\partial M$ representing $p_{\partial M}([\omega])$, clearly $\gamma \# j \omega$ is concordant to $\gamma \tilde{\omega}: \partial M \rightarrow S^{2 n+1}$. Since $\omega \in I_{n}$, there is a diffeomorphism $\Omega$ of $M$ with $\tilde{\omega}=\Omega \mid \partial M$. A lift of $\Omega$ to a diffeomorphism of $E_{M}$ can then be extended to a diffeomorphism $N \rightarrow e \cdot N$; the details are left to the reader.

Now suppose $e \in C_{2 n-1}$ and $N=(M, \gamma)$ is an $b P^{n+1}$ with $e \cdot N \approx N$. From Theorem 1,

$$
-\tau(\gamma)=\mathfrak{S}\left(N^{*}\right)=\mathfrak{S}(e \cdot N)^{*}=-\tau(\gamma \# e),
$$

so $\tau(e)=0$.

The commutative diagram (exact rows)

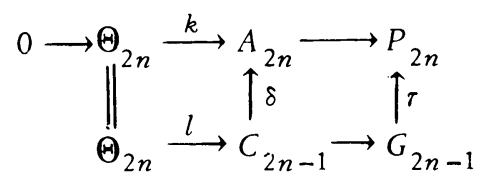

implies $e=l(\omega)$ for some $\omega \in \Theta_{2 n}$. In the diagram (upper row exact)

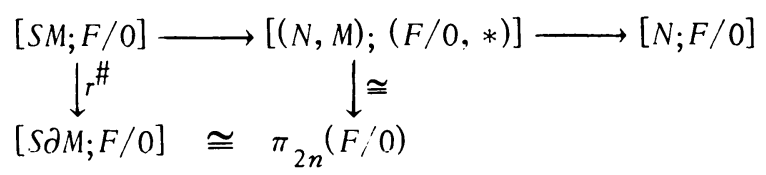

it is clear that the element of $[(N, M) ;(F / 0, *)]$ corresponding to the canonical homotopy equivalence $(e \cdot N, M) \rightarrow(N, M)$ maps to $\theta(\omega)$. Since this element goes to 0 in $[N ; F / 0], \theta(\omega)$ lies in the image of $r^{\#}$ which by Lemma 8 implies $\omega \in I(M)=$ $I_{n}$. 
6. Conjugations. If $M$ is an $b P^{n}$, and $\mu \in H^{2}(M)$ is its $c$-orientation, let $M^{-}$ be the $b P^{n}$ whose underlying manifold is $M$, and whose coorientation is $-\mu$. A conjugation of $M$ is then a diffeomorphism $f: M \rightarrow M^{-}$. Not every $b P^{n}$ admits a conjugation; for examples see [4]. We shall apply the results of $\$ 5$ to prove

Theorem 6. Let $N=(M, \gamma)$ be an $b P^{n+1}$. $N$ admits a conjugation iff there is a conjugation $f$ of $M$ such that if $\phi=f^{-1} \mid \partial M$,

(i) if $n$ is even, $\phi=$ identity,

(ii) if $n$ is odd, $\delta(\gamma \# \gamma \phi) \in I_{n}$.

Before proving this theorem, we will explain the statement (ii). If $n$ is odd $\phi$ is easily seen to be an orientation-reversing diffeomorphism of $\partial M$, and so $\gamma \# \gamma \phi$ is an embedding of $\partial M \#(-\partial M)$, which is canonically diffeomorphic to $S^{2 n-1}$. Therefore $\gamma \# \gamma \phi$ represents an element of $C_{2 n-1}$ and $\delta(\gamma \# \gamma \phi)$ is defined.

Proof of Theorem 6. Let $g$ be a conjugation of $N$, and let $b: N \rightarrow \mathbf{P}^{n+1}$ be a $c$-orientation-preserving homotopy equivalence, transverse regular on $\mathbf{P}^{n}$ with $M=$ $b^{-1}\left(\mathbf{P}^{n}\right)$. If $k$ is the usual conjugation of $\mathbf{P}^{n+1}, k \mid \mathbf{P}^{n}$ is the conjugation of $\mathbf{P}^{n}$, and so the desuspension of $N^{-}$via $k b$ is $M^{-}$. But $b g \simeq k b$, so if we let $M^{2}=$ $(h g)^{-1}\left(\mathbf{P}^{n}\right)$, Theorem 2 provides a diffeomorphism $j: N^{-} \rightarrow N^{-}$, homotopic to the identity, with $j\left(M^{2}\right)=M^{-}$. Putting $f=j g$ we see that $f$ is a conjugation of $N$ taking $M$ into $M^{-}$. In particular, $M$ admits a conjugation.

Suppose now that $f$ is a conjugation of $M$ and $\phi=f^{-1} \mid \partial M$. Then $f$ induces a diffeomorphism $F:(M, \gamma) \rightarrow\left(M^{-}, \gamma \phi\right)$. In case $n$ is even, $\left(M^{-}, \gamma \phi\right)$ is diffeomorphic to $N^{-}=\left(M^{-}, \gamma\right)$ iff $\phi \in I_{n}$; then $f$ can be replaced by a conjugation whose restriction to $\partial M$ is the identity.

If $n$ is odd, $N^{-}=\left(M^{-},-\gamma\right)$. Put $\gamma \phi=(-\gamma) \phi^{\prime}$ as embeddings of $-\partial M$ in $S^{2 n+1}$, where $\phi^{\prime}: \partial M \rightarrow \partial M$ is an orientation-preserving diffeomorphism. Let $\tilde{\phi}$ be a diffeomorphism of $S^{2 n-1}$ with $p_{\dot{\prime} M}(\tilde{\phi})$ conjugate to $\phi^{\prime}$. Then $N^{-}$is diffeomorphic to $\left(M^{-}, \gamma \phi\right)$ iff $\tilde{\phi}$ represents an element of $I_{n}$. If $j: S^{2 n-1} \rightarrow S_{\sim}^{2 n+1}$ is the standard embedding, $-\gamma \phi^{\prime}$ is concordant to $(-\gamma) \# \underset{j}{ }$. Therefore $\underset{\sim}{j}$ is concordant, as an embedding of $S^{2 n-1}$, to $\gamma \#\left(-\gamma \phi^{\prime}\right)=\gamma \# \gamma \phi$. Therefore $\tilde{\phi}$ represents $\delta(\gamma \# \gamma \phi)$ in $A_{2 n}$, and this completes the proof.

Corollary 3. Let $N=(M, \gamma)$ be an $b P^{n+1}$ which admits a conjugation, and $e \in C_{2 n-1}$. If $n$ is even, $e \cdot N$ also admits a conjugation. If $n$ is odd, $e \cdot N$ ad. mits a conjugation iff $2 \delta(e) \in I_{n}$.

7. Smoothing obstructions. Let $\exists_{n}^{\mathrm{PL}}$ be the set of PL homeomorphism classes of PL homotopy complex projective spaces. In [17], Sullivan showed $\Xi_{n}^{\mathrm{PL}}$ is naturally in 1.1 correspondence with $P_{4} \times P_{6} \times \cdots \times P_{2 n-2}$. Our methods can be simplified for the PL case to recover this classification. Define a function $k_{n}$ : $\Xi_{n}^{\mathrm{PL}} \rightarrow P_{4} \times \cdots \times P_{2 n-2}$ as follows. If $M$ is a $\mathrm{PL} b C P^{n}, M \approx\left(X, \gamma_{3}, \cdots, \gamma_{n-1}\right)$ where $X$ is an $b C P^{3}$ and $\gamma_{i}: S^{2 i-1} \rightarrow S^{2 i+1}$ is a PL embedding. An analogue of 
Theorem 4 says that $M$ is diffeomorphic to $M^{\prime}=\left(X^{\prime}, \gamma_{3}^{\prime}, \cdots, \gamma_{n-1}^{\prime}\right)$ iff $X \approx X^{\prime}$ and $\tau\left(\gamma_{i}\right)=\pi\left(\gamma_{i}^{\prime}\right), i=3, \cdots, n-1$. According to [14], there is an isomorphism $\phi: \Xi_{3} \rightarrow$ $\mathbf{Z}=P_{4}$ (notice that $\Xi_{3}=\Xi_{3}^{\mathrm{PL}}$ ); we assume the sign has been chosen to agree with [17]. Then we put $k_{n}\left(X, \gamma_{3}, \cdots, \gamma_{n-1}\right)=\left(\phi[X],-\pi\left(\gamma_{3}\right), \cdots,-\tau\left(\gamma_{n-1}\right)\right)$.

If $M$ is a PL $h C P^{n}$ and $k_{n}(M)=\left(\phi[X], m_{3}, \cdots, m_{n-1}\right), X \in \Xi_{3}, m_{j} \in P_{2 j}$, we can try to inductively choose embeddings $\gamma_{i}: \partial\left(X, \gamma_{3}, \cdots, \gamma_{i-1}\right) \rightarrow S^{2 i+1}$ with $\tau\left(\gamma_{i}\right)=-m_{i}$. If we are successful, and $\partial\left(X, \gamma_{3}, \cdots, \gamma_{n-1}\right) \approx S^{2 n-1}$, we may construct a smoothing of $M$ by attaching a disc to $\left(X, \gamma_{3}, \cdots, \gamma_{n-1}\right)$ along its boundary. The elements $\partial\left(x, \gamma_{3}, \cdots, \gamma_{i-1}\right)+\partial m_{i}$ and $\partial\left(x, \gamma_{3}, \cdots, \gamma_{n-1}\right)$ are thus obstructions to smoothing $M$. Substantially the same obstructions arise from a spectral sequence of Brumfiel [4]. Although our viewpoint is unsuitable for extensive computation, the following theorem makes the primary obstructions accessible.

Theorem 7. Let $M$ be an $b P^{n}$ such that $\partial M$ bounds a parallelisable manifold. If $n$ is even, there is a bomotopy sphere $\Sigma(M)$ such that for every embedding $\gamma$ : $\partial M \rightarrow S^{2 n+1}, \partial(M, \gamma) \approx \Sigma(M)$. If $n$ is odd and $\omega$ is a diffeomorphis $m$ of $\partial M$, $\partial(M, \gamma \omega) \approx \partial(M, \gamma) \# \eta \cdot \omega$.

$\eta$ above denotes the generator of the stable stem $\pi_{1}$, and the homotopy sphere $\eta \cdot \omega$ is constructed as in [1].

Proof. If $f$ is a diffeomorphism of $\partial M \times S^{1}$, let $w(f)$ be the element of $\pi_{1}(S F(2 n)) \cong \pi_{1}$ represented by $t \mapsto p_{1} f(x, t), x \in \partial M, t \in S^{1}$. Using an exact sequence of Browder $\left[3, \S_{5}\right]$ we see that $w(f)=0$ iff there is a diffeomorphism $\psi$ of $S^{2 n}$ such that $\int p_{\partial M \times S^{1}}(\psi)$ extends to a diffeomorphism of $\partial M \times D^{2}$.

Let $G$ denote the circle group. If $G$ acts freely on $\partial M \times D^{2}$, a trivialisation of the action on the boundary is a diffeomorphism $f$ of $\partial M \times S^{1}$ such that $f(\lambda \cdot(x, t))=(x, \lambda t)$ where $\lambda \in G, x \in \partial M, t \in S^{1}$. The particular $G$-action we have in mind is defined by the principal $G$-bundle structure on $S_{M} \approx \partial M \times D^{2}$ (compare Lemma 3); the boundary trivialisation is then, in the notation of $\$ 4$, the diffeomorphism $\sigma$. Let us assume for the moment the following lemma:

Iemma 9. If $f$ is a boundary trivialisation of the G-action on $S_{M}$, where $M$ is an $b P^{n}$, then $w(f)=n \eta$.

If $n$ is even, then $w(\sigma)=n \eta=0$. Let $\psi$ be a diffeomorphism of $S^{2 n}$ such that, setting $\psi^{\prime}=p_{\partial M \times S}(\psi), \psi^{\prime}$ extends to a diffeomorphism of $S_{M}$ onto $\partial M \times D^{2}$.

$$
-\partial(M, \gamma):=S_{M} \cup_{\Gamma}\left(S^{2 n+1}-I\left(\partial M \times B 3^{2}\right)\right),
$$

and if we let $\Sigma(M)$ be the homotopy sphere $D^{2 n+1} \cup_{\psi} D^{2 n+1}$,

$$
-\partial(M, \gamma) \# \Sigma(M) \approx s_{M} \cup_{\Gamma \curvearrowleft \psi^{\prime}}\left(s^{2 n+1}-\Gamma\left(\partial M \times B^{2}\right)\right) .
$$


Since $\Gamma \Sigma \psi^{\prime}$ extends over $S_{M},-\partial(M, \gamma) \# \sigma(M) \approx S^{2 n+1}$, or $\partial(M, \gamma) \approx \Sigma(M)$.

We now consider the case where $n$ is odd. Let $\phi$ be an essential circle action on $\partial M$; then $w(\phi)=\eta$ (cf. Lemma 8 ), so that if $f$ is the diffeomorphism of $\partial M \times S^{1}$ defined by $f(x, t)=(\phi(t, x), t), x \in M, t \in S^{1}$, then $w(f)=\eta$. If $\omega$ is a diffeomorphism of $\partial M$, put $g_{\omega}=\omega \times$ id: $\partial M \times S^{1} \rightarrow \partial M \times S^{1}$. Then, by following De Sapio [s], $\eta \cdot \omega$ can be shown to be the obstruction to extending the diffeomorphism $f^{-1} g_{\omega} f$ of $\partial M \times S^{1}$ to a diffeomorphism of $\partial M \times D^{2}$.

Now let $U \approx \partial M \times[1 / 2,1]$ be a collar of $\partial M$ in $M$, and $M_{1}=\mathrm{Cl}(M-U)$. Choose a diffeomorphism $b: \partial M \times D^{2} \rightarrow S_{M_{1}}$; then

$$
\begin{aligned}
-\partial(M, \gamma) \# \eta \cdot \omega & \approx S_{M} U_{b g_{\omega} f b^{-1}} S_{U} \cup_{\Gamma \sigma}\left(S^{2 n+1}-\Gamma\left(\partial M \times B^{2}\right)\right) \\
& \approx-\partial(M, \gamma \omega) .
\end{aligned}
$$

Since $2 \eta \cdot \omega \approx S^{2 n+1}$ this completes the proof of Theorem 7 .

Proof of Lemma 9. I am indebted to Professor Bredon for a suggestion concerning this proof. Denote by $\phi$ the action of $G$ on $S_{M}$ induced by the principal $G$-bundle structure, so $\phi: G \times S_{M} \rightarrow S_{M}$. Let $\psi: G \times \partial M \times D^{2} \rightarrow \partial M \times D^{2}$ be the action defined by $\psi(\lambda, x, w)=(x, \lambda w)$, where $\lambda \in G, x \in \partial M, w \in D^{2}$ and the action of $G$ on $D^{2}$ is the usual one. To simplify our notation, we identify the manifolds $S_{M}$ and $\partial M \times D^{2} ;$ in doing so we require that the composition

$$
\partial M \stackrel{(\mathrm{id}, 1)}{\longrightarrow} \partial M \times S^{1} \stackrel{f}{\longrightarrow} \partial M \times S^{1} \stackrel{\text { proi }}{\longrightarrow} \partial M
$$

be homotopic to the identity, and $p_{2} f(\partial M \times\{1\})=\{1\}$. It is clear that, as maps of $\partial M \times S^{1}, f$ is homotopic to $f^{-1}$. Consider the diagram

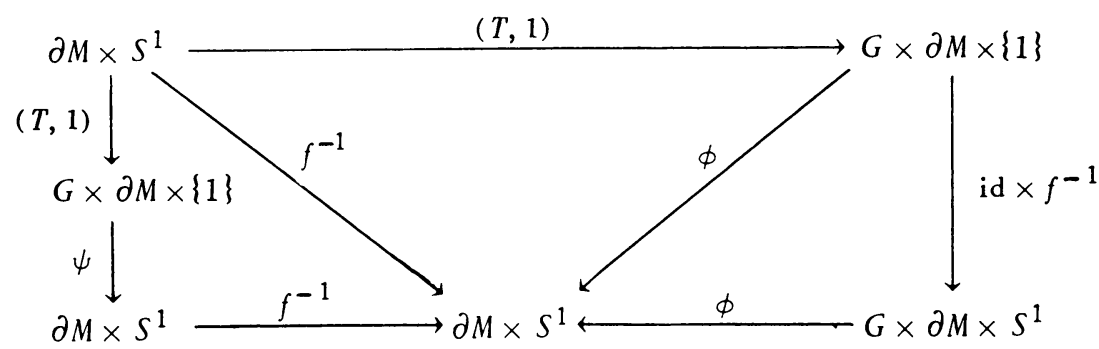

The triangle on the right homotopy commutes because of the above requirements, and the left triangle is easily seen to be commutative. Furthermore, the entire rectangle commutes because $f^{-1}$ is equivariant from the action $\psi$ to the action $\phi$. Therefore the middle triangle is homotopy commutative: $\phi(T, 1) \simeq f^{-1} \simeq f$. But the action $\phi$ on $\partial M \times D^{2}$ is equivariantly homotopy equivalent to the free $G$-action $\phi_{0}$ on $S_{\mathbf{p} n}=S^{2 n-1} \times D^{2}$; it is easily seen that $w\left(\phi_{0}(T, 1)\right)=n \eta$ and this completes the proof. 
The following application of Theorem 7 may be of interest. For every embedding $\gamma: S^{4 k-1} \rightarrow S^{4 k+1}, \partial\left(\mathbf{P}^{2 k}, \gamma\right) \approx S^{4 k+1}$; therefore the PL $b C P^{2 k+1}, M$, with $k_{2 k+1}(M)=(0, \cdots, 0, m)$ is smoothable iff $\partial m$ is diffeomorphic to $S^{4 k-1}$. This observation leads to the solution of a problem proposed by K. Wang at the 1971 Conference on Transformation Groups at Amherst: to show that in every dimension $4 k+3$ there are infinitely many free smooth circle group actions on homotopy spheres which do not extend to free $S^{3}$ actions. Using [19], we notice that if $m \neq 0, M$ is the orbit space of such a free circle action.

\section{REFERENCES}

1. G. E. Bredon, $A \pi_{*}$-module structure for $\Theta_{*}$ and applications to transformation groups, Ann. of Math (2) 86 (1967), 434-448. MR $36 \# 4570$.

2. E. Brieskorn and A. Van de Ven, Some complex structures on products of homotopy spheres, Topology 7 (1968), 389-393. MR 38 \#1682.

3. W. Browder, Diffeomorphisms of 1-connected manifolds, Trans. Amer. Math. Soc. 128 (1967), 155-163. MR $35 \# 3681$.

4. G. Brumfiel, Differentiable $S^{1}$ actions on homotopy spheres, Berkeley (preprint).

5. R. De Sapio, Differential structures on a product of spheres. II, Ann. of Math. (2) 89 (1969), 305-313. MR $39 \# 7611$.

6. A. Haefliger, Sphères d'homotopie noueés, Séminaire Bourbaki 1964/65, Exposé 280, Benjamin, New York, 1966. MR 33 \#54201.

7. - Differentiable embeddings of $S^{n}$ in $S^{n+q}$ for $q>2$, Ann. of Math. (2) 83 (1966), 402-436. MR 34 \#2024.

8. M. W. Hirsch, Obstruction theories for smoothing manifolds and maps, Bull. Amer. Math. Soc. 69 (1963), 352-356. MR $26 \# 6980$.

9. W.-C. Hsiang, A note on free differentiable actions of $S^{1}$ and $S^{3}$ on homotopy spheres, Ann. of Math. (2) 83 (1966), 266-272. MR 33 \#731.

10. W.-C. Hsiang and W.-Y. Hsiang, Some free differentiable sections of $S^{1}$ and $S^{3}$ on 11-spheres, Quart. J. Math. Oxford Ser. (2) 15 (1964), 371-374. MR 30 \#3479.

11. M. A. Kervaire, On higher dimensional knots, Differential and Combinatorial Topology (A Sympos. in Honor of Marston Morse), Princeton Univ. Press, Princeton, N. J., 1965, pp. 105-119. MR $31 \# 2732$.

12. M. A. Kervaire and J. W. Milnor, Groups of homotopy spheres. I, Ann. of Math. (2) 77 (1963), 504-537. MR 26 \#5584.

13. J. Levine, Knot cobordism groups in codimension two, Comment. Math. Helv. 44 (1969), 229-244.

14. D. Montgomery and C. T. Yang, Differentiable actions on homotopy seven spheres, Trans. Amer. Math. Soc. 122 (1966), 480-498. MR $34 \# 820$.

15. - Free differentiable actions on homotopy spheres, Proc. Conf. on Transformation Groups (New Orleans, La., 1967), Springer, New York, 1968, pp. 175-192. MR $39 \# 6354$.

16. R. Schultz, The nonexistence of free $S^{1}$ actions on some homotopy spheres, Proc. Amer. Math. Soc. 27 (1971), 595-597. MR $42 \# 6866$. 
17. D. Sullivan, Triangulating and smoothing homotopy equivalences and homeomorphisms, Geometric Topology Seminar Notes, Princeton University, Princeton, N. J., 1967.

18. C. T. C. Wall, An extension of results of Novikov and Browder, Amer. J. Math. 88 (1966), 20-32. MR 35 \#3691.

19. B. Conrad, Extending free circle actions on spheres to $S^{3}$ actions, Proc. Amer. Math. Soc. 27 (1971), 168-174. MR $43 \# 1224$.

DEPARTMENT OF MATHEMATICS, TEMPLE UNIVERSITY, PHILADELPHIA, PENNSYLVANIA 19122 